\title{
INVESTIGATION OF BOILING IN HIGH-POWER LED COOLING SYSTEM
}

\author{
Evgeniy E. Shatskiy ${ }^{1,2, *}$ \\ ${ }^{1}$ Kutateladze Institute of Thermophysics SB RAS, 630090 Novosibirsk, Russia \\ ${ }^{2}$ Novosibirsk state University, 630090 Novosibirsk, Russia
}

\begin{abstract}
The technology of solid-state light source is associated with the future of a number of sectors of the economy. The duration of failure-free operation, optical radiation power and other output characteristics of the LEDs are closely linked with p-n junction temperature, which makes the development of the cooling systems an important step in creating LED systems. In this work, we have created a new experimental setup for studying heat transfer from a local heat source, modelling the LED chip. The boiling heat transfer on the local heaters with a diameter of 5 and 10 $\mathrm{mm}$ has been investigated at the saturation temperature in bulk liquid. It is shown that on the finned surfaces the overheating relative to the saturation temperature in comparison with a smooth surface decreases up to one and a half times for the heater with a diameter of $10 \mathrm{~mm}$, and up to three times for the heater with a diameter of $5 \mathrm{~mm}$. There is up to two times increase in heat transfer coefficient on finned surfaces as compared to the smooth ones. For finned surfaces on the heater with a diameter of $1 \mathrm{~mm}$ the surface overheating relative to the saturation temperature decreases four times. More than three times increase is observed for the heat transfer coefficient on finned surfaces as compared to the smooth ones.
\end{abstract}

\section{Introduction}

Cooling of heat-emitting surfaces in different technological applications is an important problem, which can be solved by such processes as film flows [1, 2], etc. In our days the problem of creating an effective compact cooling system for powerful LEDs whose efficiency and durability are highly dependent on the efficiency of the power removal remains unsolved.

Study of evaporation mechanism on the surfaces of small size (SSS) was started in [3]. A review of works on heat transfer at evaporation on SSS may be found in [4]. In the literature, there is a dependencies for the critical heat flux density for each type of SSS, having the general form:

$$
q_{c r} / q_{c r \infty}=k\left(d_{s} / l_{\sigma}\right)^{n}
$$

\footnotetext{
* Corresponding author: shatskiy.itp@gmail.com
} 
but differing in correlation multipliers; and $d_{s}$ is the diameter of the heating surface. Here

$$
q_{c r o \infty}=0.13 r_{l v} \rho_{v}\left(\frac{\sigma\left(\rho-\rho_{v}\right) g}{\rho_{v}^{2}}\right)^{0.25}
$$

$r_{l v}$ is the latent heat of vaporization, $\rho_{\mathrm{v}}$ is the vapor density, $\rho$ is the fluid density, $\sigma$ is the surface tension, and $\mathrm{g}$ is the gravity acceleration.

For locally heated horizontal plates $\mathrm{k}=3.4, \mathrm{n}=-0.5$ [4], for horizontal cylinders $\mathrm{k}=1.4$ [5], and for spheres $\mathrm{k}=2.5$ [5]. The reason for such significant effect of the size of SSS on $\mathrm{q}_{\mathrm{cr}}$ is connected with the feature of hydrodynamic situation that occurs around the surface of small size (SSS).

\section{Experimental setup}

To study the heat transfer processes that occur during cooling of LED equipment in the boiling liquid, a new experimental setup was created. Schematic diagram of the experimental setup is shown in figure 1 .

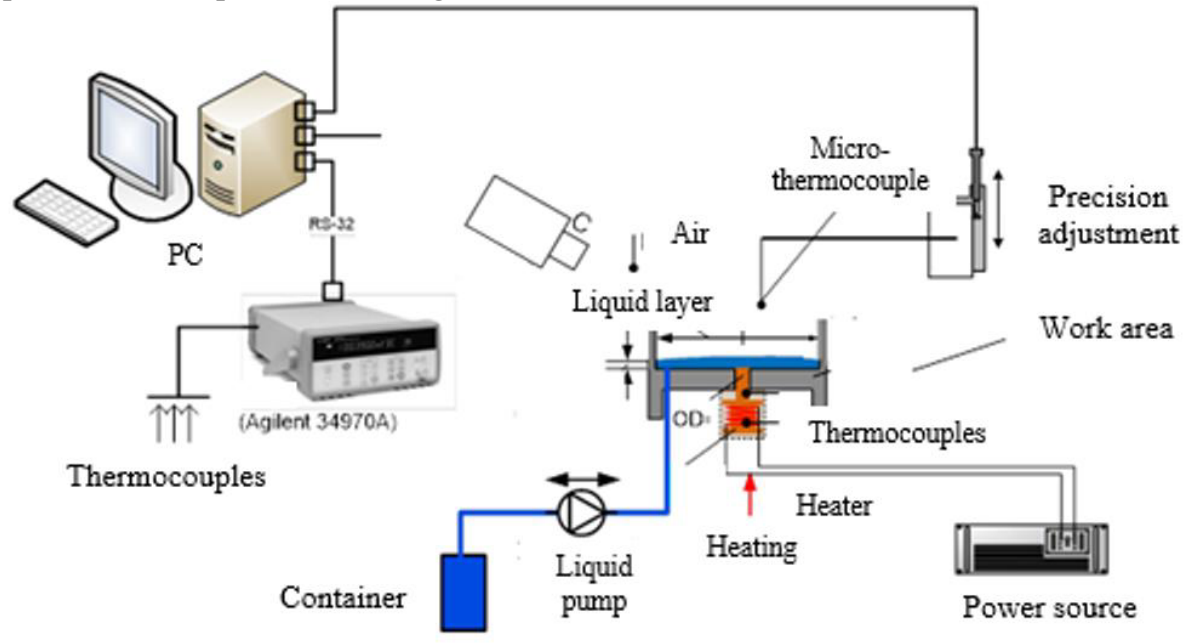

Fig. 1. Scheme of experimental setup.

The working fluid is supplied to the work area by means of a peristaltic pump. There the horizontal layer of liquid is formed. The thickness varies depending on the operating parameters of the experiment. In the center of the layer from the side of the substrate there is local heating performed by a copper heater. The power of the heating element is controlled by the power source. The temperature of the heater, the fluid and the medium is measured by a number of thermocouples. The data from thermocouples is collected using a control and measuring system, and the temperature measurement precision is $0.1{ }^{0} \mathrm{C}$.

\section{Results and discussion}

Figure 2 presents a comparison of the obtained experimental data on the critical heat flux density with the calculated dependence (1) of [4] and results of work [6]. The figure shows that the density of the critical heat flux in a bulk liquid for heaters with $\mathrm{D}=1.6$ and $5 \mathrm{~mm}$ is in good agreement with the dependence (1) and experimental data for vertical heaters. The 
heater size has a significant influence on the critical heat flux density (CHFD). The radial finning of the heater has no effect on CHFD, and the decrease of the layer height and the flow rate of the supplied liquid leads to a significant decrease in CHFD. The value of CHFD for $\mathrm{D}=1 \mathrm{~mm}$ is higher than the estimated one. Moreover, the highest increase was achieved by using finned surface.

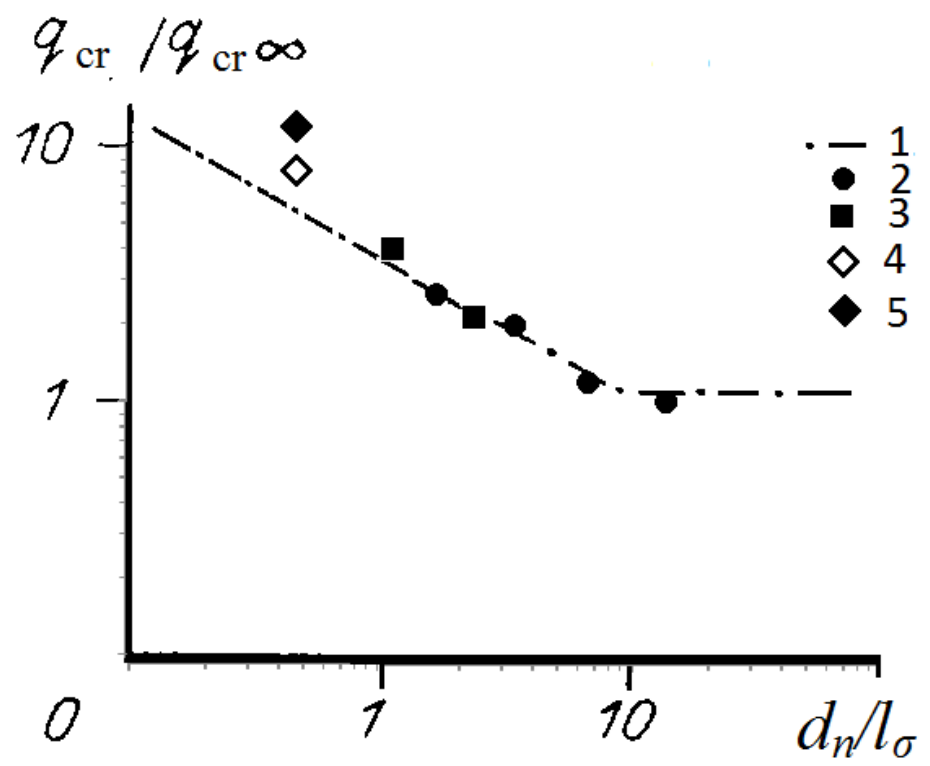

Fig. 2. - The dependence of CHFD on the size of the heater. 1 - calculation by formula (1) from [4], 2 - data from [6], $3-\mathrm{D}=1.6$ and $5 \mathrm{~mm}, 4-\mathrm{D}=1 \mathrm{~mm}, 5-\mathrm{D}=1 \mathrm{~mm}$ with finning.

Experiments on the heaters with $5 \mathrm{~mm}$ diameter and smooth and finned surfaces have showed that for the finned surfaces the overheating relative to the saturation temperature decreases up to three times. Finning on the heater with $1 \mathrm{~mm}$ diameter decreases the overheating relative to the saturation temperature more than four times. Figure 3 shows the effect of the liquid temperature around the heat exchange surface on the dependence of the heat flux density for saturation temperature (points 1-3) and at a temperature of $25-30{ }^{\circ} \mathrm{C}$. Such regimes are possible in thermosiphons and heat pipes, if the regime of subcooling of condensed liquid is realized. It is seen that in this case, the temperature of the LED chip model is significantly reduced. It is shown that for the heat flux densities up to $1000 \mathrm{~W} / \mathrm{cm}^{2}$ the temperature of the thermal model of the LED chip does not exceed $120^{\circ} \mathrm{C}$. 


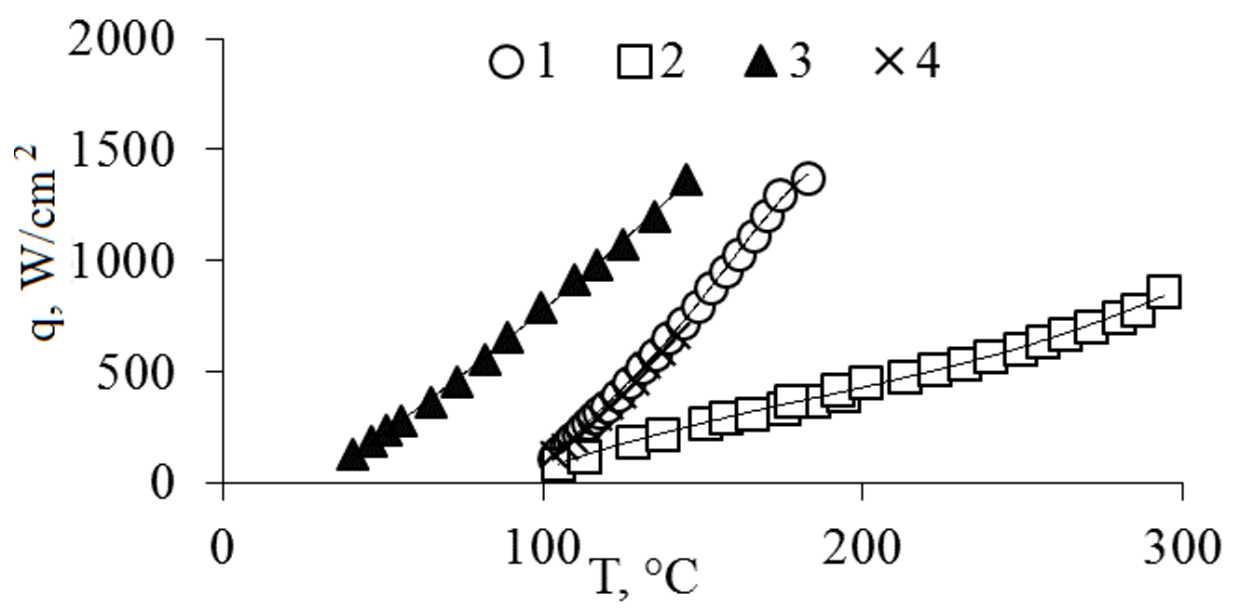

Fig. 3. - The dependence of the removed heat flux density on temperature, $D=1 \mathrm{~mm}$. 1 - large fins, 2 - smooth surface, 3 - nozzle with small fins, $\mathrm{T}=25-30^{\circ} \mathrm{C}, 4-$ small fins under saturated conditions.

\section{Acknowledgments}

The work was supported by the RF Ministry of Education and Science (Agreement No. 14.604.21.0053, project ID RFMEFI60414X0053).

\section{References}

1. E.A. Chinnov, J. Heat Transfer 138 (2016)

2. O.A. Kabov, D.V. Zaitsev, V.V. Cheverda and A. Bar-Cohen, Exp. Therm Fluid Sci. 35, $5(2011)$

3. F. D. Moor, R. B. Mesler, AlChE J. 7, 4 (1961)

4. V.I. Tolubinskiy, V.A. Antonenko, G.R. Kudritskiy, Yu.N. Ostrovskiy, Industrial Heat Engineering 9, 4 (1987)

5. D. Linard, B. Dir, Teploperedacha 2 (1973)

6. E.A. Chinnov, A.V. Dyatlov, D.N. Kravchenko, J. Siberian Physico-Technical 5 (1992) 